\title{
Estudio histórico-epistemológico del trabajo de Amadeo Avogadro
}

\author{
Study historical epistemological of the Avogadro's work \\ Katerin Farieta Bonilla ${ }^{1}$ y Rómulo Gallego Badillo² \\ ${ }^{1}$ Estudiantes Maestría en Docencia de la Química. Universidad Pedagógica Nacional. ${ }^{2}$ Profesor \\ Universidad Pedagógica Nacional. dq12 kfarieta@pedagogica.edu.co rgallego@pedagogica.edu.co
}

\section{Resumen}

En este artículo presenta un análisis del trabajo de Amadeo Avogadro desde una perspectiva histórica-epistemológica, empezando con la reconstrucción de las teorías formuladas por Dalton y Gay Lussac, como antecedentes inmediatos del ensayo de Avogadro. Finalmente, se presenta la contribución de Cannizzaro para la aceptación de la teoría de Avogadro.

\section{Palabras Clave}

Análisis histórico, análisis epistemológico, Amadeo Avogadro.

\section{Abstract}

This paper presents an analysis of Avogadro's work since an historical-epistemological perspective, beginning with a reconstruction of the theories formulated by Dalton and Gay Lussacc as immediate antecedents of the essay of Avogadro. Finally, is presented the contribution of Canizzaro to the acceptation of Avogadro's theory.

Keywords

Historical analysis, epistemological analysis, Amadeo Avogadro

\section{Introducción}

El estudio histórico-epistemológico de una teoría resulta de gran importancia para los especialistas en el campo de la didáctica de las ciencias experimentales, ya que ha contribuido a la construcción de los diferentes modelos de enseñanza como aprendizaje por descubrimiento, el cambio conceptual y el tratamiento de situaciones problemicas (Gil 1993). A demás, dicho enfoque contribuye de manera sustancial a la investigación sobre la formación de docentes, el análisis de libros de texto y la transposición didáctica, entre otros.

El presente trabajo busca dejar de lado las versiones historicistas que dominan las dases de ciencias. Para tal fin, se propone abordar la historia desde una epistemología inductivista (Bacón, 1949) y desde la versión de la química como modelo cient́fico. El rumbo trazado implica analizar los textos originales de John Dalton (1808), Amadeo Avogadro (1811) y Cannizzaro (1858). Teniendo en auenta el contexto histórico de los escritos mencionados, la epistemología inductivista de Bacón y la propuesta de Kuhn permiten vislumbrar el proceso, cient́fico y social, a partir del aual se consolidaron las teorías atómicas y moleculares. 


\section{Construcción de la hipótesis de Amadeo Avogradro}

El trabajo de Avogadro contó con los trabajos de John Dalton acerca del peso relativo de las partículas, posteriormente denominado peso atómico, como antecedente. Dalton tomó como punto de partida los estudios meteorológicos y su análisis de las propiedades de los gases, desde una posición inductivista de la ciencia. Por otro lado, aparecen los trabajos de Gay Lussacc (1809), quien desarrollańa la ley de combinación de los gases y la relación con sus masas relativas. A continuación se profundiza en la caracterización de los trabajos de Dalton y Gay Lussacc. Resaltando el enfoque epistemológico a partir del cual desarrollaron sus propuestas.

\section{Dalton y sus reglas de Máxima Simplicidad}

John Dalton partió de los diferentes datos obtenidos en los análisis elementales de los compuestos desarrollados en la época. Es necesario anotar las dificultades experimentales que implicaban la obtención de una gran variedad de datos. Por ejemplo, en la molécula de agua asume los valores de 5,5 y 7 partes de oxigeno frente a 1 de hidrogeno, a la vez que asume el valor del hidrogeno como de referencia para calcular los pesos atómicos de los demás elementos. El problema radica en que los valores la molécula de agua no se encuentra sólidamente respaldada por la experimentación. Dejando de lado las dificultades experimentales, se presenta como un segundo problema el conocimiento de la información microscópica de los compuestos, debido a que la teoría de Dalton no reconoce la relación entre los átomos de un compuesto. Por ejemplo en el caso antes mencionado, al descomponerse el agua se obtiene gas de hidrogeno y gas de oxigeno. De lo anterior se plantea la pregunta ¿Cómo obtener la relación de los átomos de un compuesto?

Dalton, plantea como hipótesis un conjunto de reglas que denomino "reglas de máxima simplicidad", que enunció en su libro "A New system of chemical phylosophy" de la siguiente manera:

$>$ Cuando puede obtenerse un solo compuesto de dos cuerpos, se le llamańa binario, a menos que alguna causa indique lo contrario.

$>$ Cuando se obtienen dos compuestos de la combinación de dos cuerpos, se les supondrá uno binario y otro temario.

$>$ Cuando se obtienen tres compuestos, podemos esperar que uno de ellos sea binario y los otros dos temarios

$>$ Cuando se observan cuatro compuestos, deberíamos esperar uno binario, dos ternarios y uno cuatemario, etc.

$>$ Un compuesto binario debeńa ser siempre espećficamente más pesado que la mera mezda de sus dos ingredientes.

$>$ Un compuesto temario debería ser espeáficamente más pesado que la mezda de un binario y uno simple que, de combinarse lo constituirían.

$>\quad$ Las reglas y observaciones anteriores se aplican igualmente cuando se combinan dos cuerpos.

Partiendo de estas reglas, Dalton conduye que el agua es un compuesto binario de hidrógeno y oxígeno y los pesos relativos de los átomos elementales están aproximadamente 1:7.

Para dar otro ejemplo, para los óxidos de carbono Dalton distinguía dos compuestos: uno binario (el monóxido de carbono) y uno temario (el dióxido de carbono), obedeciendo a la segunda de sus reglas.

Desde la versión de la química como modelo científico se muestra como Dalton construye su propio modelo, contrario a la versión positivista de la historia, sin ninguna base empírica, 
totalmente hipotética (Themard, 1777-1857), aunque necesaria para la obtención de los pesos atómicos.

\section{Gay Lussacc y los volúmenes de Combinación}

Gay Lussac pretendía dar a conocer nuevas propiedades de los gases mostrando: primero, que las proporciones se combinan entre sí en proporciones muy simples; y segundo, la contracción del volumen frente a la presión sigue un patrón determinado. Para esto parte de las investigaciones realizadas por Proust, con la idea de la existencia de dos grados de oxidación; Berthollet, los compuestos se forman de relaciones muy variables; y Dalton, en lo que se refiere a sus leyes de combinación.

Para mostrar su primera propuesta realizo combinaciones de gases de ácidos (muriático, fluorhídrico y carbónico), con gas álcali (amoniaco) para formar sales neutras, llegando a conduir que volúmenes exactos e iguales de acido se combinan con volúmenes exactos e iguales de álcali.

En síntesis, Gay Lussacc muestra en su memoria "memoires de la Société d'Arcueil, tomo II" que las combinaciones entre gases se efectúan según relaciones de volúmenes simples y que auando el resultado de la combinación es un gas, su volumen también está en relación simple con el de sus componentes. Pero las relaciones de las cantidades de estas sustancias dependían a su vez de del numero de moléculas que se combinan. Al respecto Gay Lussacc conduye que los gases, independientemente de las proporciones en las que se puedan combinar, dan siempre lugar a compuestos ayyos elementos, medidos en volumen, son siempre múltiplos uno del otro.

\section{La propuesta de Amadeo Avogadro}

A partir de estos estudios Avogadro comienza su "Ensayo de una manera de determinar las masas relativas de las moléculas elementales de los auerpos y las proporciones según las cuales ellas entran en estas combinaciones". Avogadro fue influenciado por la química francesa, debido al contexto geográfico en que desarrolló su trabajo. Por lo tanto, no resulta difícil entender la razón por la cual su obra se encuentra influenciada en mayor medida por los trabajos de su cotenáneo Gay Lussacc, en comparación con los aportes de Dalton, quien residía en Inglaterra.

Vale la pena mencionar la dificultad que se presenta de la lectura del ensayo de Avogadro, debido al lenguaje empleado, ya que no existía una dara diferencia entre lo que actualmente se conoce como átomos y moléculas. Avogadro empleo la palabra molécula refiriéndose a lo que Dalton definía como átomo, cuestión que refleja las diferencias entre los científicos franceses e ingleses de la época.

Según Avogadro, las conclusiones de Gay Lussacc acerca de la las combinaciones de los gases dan lugar siempre a relaciones muy simples de volúmenes. Avogadro admite la existencia de relaciones muy simples entre los volúmenes de las sustancias gaseosas y el número de moléculas simples o compuestas que los forman. De allí se plantea su hipótesis, según la cual "suponer que el número de moléculas integrantes en unos gases aualquiera es siempre la misma en un volumen igual o es siempre proporcional a los volúmenes. En efecto si se supusiera que el que el numero de moléculas contenidas en un volumen dado fuera diferente para los diferentes gases, sería casi imposible pensar que la ley que rige las distancias de las moléculas pudiera dar, en todos los casos, relaciones tan simples como los hechos que acabamos de citar nos obligan a admitir entre el volumen y el numero de moléculas. " Avogadro plantea su hipótesis a partir de la experiencia de Gay Lussacc, siguiendo el método inductivista, dado que parte de la observación de los hechos (Bacon, 1620). 
Partiendo de esta hipótesis, Avogadro propone una forma de determinar fácilmente las masas relativas de las moléculas de los cuerpos que pueden ser gaseosas y el numero relativo de de estas moléculas en las combinaciones. Es decir, si volúmenes iguales de gases contenían igual número de partículas, las relaciones de las densidades de los gases debían ser igual a la relación de las masas de dichas partículas.

Avogadro describe las experiencias de Gay Lussacc acerca de los volúmenes. Según éste último, la relación entre el oxigeno y el hidrogeno en la molécula de agua era de 1 a 2, lo que permite concluir que la relación de masas de dichos elementos en la molécula es, igualmente, de 1 a 2 . Esta suposición era contraria a la de Dalton y sus reglas de máxima simplicidad, en donde sus cálaulos lo llevaban a afirmar que la masa del oxigeno con respecto al Hidrogeno es de 6 a 1; mientras que Avogadro, bajo sus supuestos, mostraba una relación de 15 a 1.

Sin embargo, las suposiciones de Avogadro no estaban libres de problemas. Al aceptar la relación de 2 a 1, el peso de una molécula de agua seria igual a 17. Pero los datos experimentales de densidades de vapores mostraban que el peso de la molécula de agua era 8,537 , esto es, la mitad del valor obtenido por la suma de los pesos de las moléculas de hidrogeno y oxigeno.

Para solucionar este problema, Avogadro plantea que "basta suponer que la moléculas constituyentes de un gas simple cualquiera (...) no están formadas por una sola molécula elemental, sino que resultan de un cierto numero de estas moléculas reunidas en una sola atracción y que cuando las moléculas de otra sustancia deben unirse a aquellas para formar moléculas compuestas, la molécula integrante que debería resultar de ellas se divide en dos o más partes o moléculas integrantes formadas de la mitad, la cuarta parte, etc. De esta segunda sustancia; de modo que el número de moléculas integrantes del compuesto llega a ser el doble, el auádruple, etc. De lo que debería ser sin esta división, y el valor necesario para satisfacer el volumen del gas que resulta". Tal y como lo describen Muñoz y Bertomeuen, "en este confuso párrafo Avogadro sugiere que cuando se forma una compuesto, las moléculas se dividen en dos o mas partes de modo que cada una de ellas contiene medias moléculas, cuartos de moléculas etc. De los reactivos de los que dan lugar a su formación". Así las cosas, el agua, al formarse, se dividía en dos, de modo que estańa constituida por una demi-molecula de oxigeno y una de hidrogeno, lo que concordaba con la hipótesis antes mencionada. El amoniaco, por su parte, se formaría a partir de de tres moléculas de hidrogeno por una de nitrógeno y la molécula constituida se dividía en dos.

\section{Stanislao Cannizzaro}

Medio siglo después de la investigación de Avogadro, Stanislao Cannizzaro es le otorga un lugar central en su "LETTER OF PROFESSOR STANISLAO CANNIZZARO", donde confirma la hipótesis del químico francés, igual que las propuestas de Ampere y Dumas, en lo que tiene que ver con el estudio de sustancias gaseosas.

Debido a la gran confusión que existía en la época a cerca de la teoría de pesos atómicos y a la gran aceptación de la teoría atómica de Dalton, Kekulé convocó a la primera reunión científica intemacional de la historia. El Primer congreso Internacional de Química (y tuvo lugar en 1860 en Karlsruhe). En dicho evento, con el fin de convencer a sus lectores, Cannizzaro realizó un examen histórico de las teorías químicas. En una serie de conferencias demostró como a partir de la los trabajos de Gay- Lussac surge la hipótesis de Avogadro y la de Ampere, en donde se realiza la diferenciación entre átomos y moléculas y la deducción de los pesos atómicos, y además expuso como las teorías que especifica no tiene contradicciones. En suma, Cannizzaro echó abajo las razones por las cuales la teoría de Avogadro no era aceptada por los químicos de la época. 
Desde la postura epistemológica de Kuhn (1962), se puede decir las discusiones entre la comunidad de especialistas reunida en el primer Congreso Internacional de Química determinó la revolución científica que modificó el paradigma sobre la diferenciación de átomos y moléculas, acabando con la hegemonía de la propuesta de Dalton.

\section{Conclusión}

El enfoque inductivo de la reconstrucción realizada permite entrever el proceso a partir del cual se desarrolló la teońa atómica molecular. La formulación de la tesis de Avogadro se entiende, siguiendo la lógica inductivista, como una síntesis de las propuestas de Dalton y Gay Lussacc, sin las cuales la propuesta de Avogadro no puede ser del todo comprendida, y menos enseñada. A mismo tiempo, la reconstrucción presentada pretende señalar que si bien la aceptación general de la hipótesis de Avogadro fue posible gracias a su solidez empírica, fueron las discusiones de la comunidad científica, resumidas en este caso por el primer Congreso Internacional de Química, de las que se conduyó la validez teórica y experimental de la teoría atómica molecular del químico francés.

\section{Bibliografía}

Avogadro, A (1811). Essay on a Manner of Determining the Relative Masses of the Elementary Molecules of Bodies, and the Proportions in Which They Enter into These Compounds. Journal de Physique, de Chimie et d'Histoire naturelle. 73 (4), 58-76.

Dalton, J. (1808). A New System of Chemical Philosophy. Parte I. Manchester, R. Bidkeerstaff.

Cannizzaro, S. (1858). "Sunto di corso di filosofía chimica fatto nella Reale Universitá di Genova". Nuovo Cimento, 7, 321-366.

Gil, D. (1993). Contribución de la historia y de la filosofía de las ciencias al desarrollo de las ciencias al desarrollo de un modelo de enseñanza aprendizaje como investigación. Enseñanza de las ciencias, 11 (2), 197-212 197

Kuhn T. S. (1972). La estructura de las revoluciones científicas México. Fondo de Cultura Económica. 